\title{
Padrão alimentar de risco para as doenças crônicas não transmissíveis e sua associação com a gordura corporal - uma revisão sistemática
}

\author{
Dietary risk patterns for non-communicable chronic diseases \\ and their association with body fat - a systematic review
}

Edynara Cristiane de Castro Azevedo ${ }^{1}$

Alcides da Silva Diniz ${ }^{1}$

Jailma Santos Monteiro ${ }^{1}$

Poliana Coelho Cabral ${ }^{1}$

${ }^{1}$ Programa de PósGraduação em Nutrição, Universidade Federal de Pernambuco. R. Prof. Moraes 1235, Cidade Universitária. 50.670-901 Recife PE Brasil. edy_azevedo@hotmail.com

\begin{abstract}
The article seeks to review studies on unhealthy eating patterns for chronic non-communicable diseases (NCD) and its association with body fat in adults, published between 2005 and 2012. The articles were systematically reviewed by two independent researchers in Medline, Lilacs, and Scielo using the following key words: food consumption, chronic diseases, anthropometry, body fat, and adults. Eight studies met the inclusion criteria, all conducted on the Brazilian population. The prevalence of overweight and $a b$ dominal fat ranged from $38.6 \%$ to $51.3 \%$ and $23.4 \%$ to $43.1 \%$, respectively. In the majority of studies, fruit and vegetable consumption was below the recommended level. A significant association between food patterns and anthropometric profile was found in five studies. The variations in the prevalence and in the results of the studies indicate the need for standardization of data collection instruments and methods used, as well as conducting studies with a more appropriate design. The high prevalence of overweight and low consumption of food meeting healthy eating standards indicates the need for intervention measures. Key words Food consumption, Adults, Anthropometry, Systematic review, Chronic non-communicable diseases, Body fat
\end{abstract}

Resumo O objetivo do presente artigo é revisar estudos sobre padrão alimentar de risco para as doenças crônicas não transmissíveis (DCNT) e sua associação com a gordura corporal em adultos, publicados entre os anos de 2005 e 2012. Os artigos foram revisados sistematicamente de forma independente por 2 pesquisadores, utilizando os descritores: food consumption, chronics diseases, anthropometry, body fat e adults, nas bases de dados eletrônicas Medline, Lilacs e Scielo. Foram identificados 8 estudos que atenderam aos critérios de inclusão, todos realizados na população brasileira. A prevalência do excesso de peso e de gordura abdominal oscilou de 38,6\% a 51,3\% e $23,4 \%$ a $43,1 \%$, respectivamente. Na maioria dos estudos, o consumo de frutas, legumes e verduras esteve abaixo do recomendado. Foi encontrada associação significativa entre padrões alimentares e perfil antropométrico em 5 estudos. As variações nas prevalências e nos resultados dos estudos indicam a necessidade de padronização dos instrumentos de coleta e métodos utilizados e a realização de estudos com delineamentos mais apropriados. As altas prevalências de excesso de peso e de baixo consumo de alimentos marcadores de padrões alimentares saudáveis indicam a necessidade de medidas de intervenção.

Palavras-chave Consumo alimentar, Adultos, Antropometria, Revisão sistemática, Doenças crônicas não transmissíveis, Gordura corporal 


\section{Introdução}

As doenças crônicas não transmissíveis (DCNT) constituem um dos maiores problemas de saúde pública no mundo ${ }^{1}$. No Brasil, cerca de $70 \%$ das causas de mortes na população adulta são devidas a essas doenças ${ }^{2}$. Esse padrão de morbimortalidade tem sido atribuído ao processo de transição epidemiológica, caracterizado pelo incremento das DCNT e declínio das doenças infecciosas, pari passu ao advento da transição nutricional, marcada pelo aumento do consumo de alimentos com alta densidade energética e diminuição do consumo de alimentos ricos em fibras". Essas mudanças no padrão alimentar e no estilo de vida imprimiram um aumento significativo na prevalência de excesso de peso e obesidade, sendo que esta tem sido considerada como um dos principais fatores de risco para as DCNT. Esses achados são confirmados pela Pesquisa de Orçamentos Familiares (POF) ${ }^{5}$, que constatou uma prevalência de $50 \%$ de excesso de peso na população adulta brasileira, baixo consumo de alimentos ricos em fibras, como frutas, verduras e leguminosas e alto consumo de alimentos ricos em gordura saturada, açúcar e sal. Dados recentes $^{6}$ mostram que menos de $1 / 4$ da população possui consumo recomendado de frutas e hortaliças, o que reflete a baixa qualidade da dieta do brasileiro.[falta a citação 3 entre a 2 e a 4]

Dentre as DCNT, destacam-se o diabetes melittus, as doenças cardiovasculares, alguns tipos de cânceres e a obesidade ${ }^{1}$. A inatividade física, o alcoolismo, o tabagismo, o consumo de alimentos pobres em fibras e vitaminas antioxidantes e ricos em gorduras saturadas, gordura trans e açúcares simples têm sido identificados como preditores independentes dessas enfermidades ${ }^{1,7}$.

A importância da obesidade dentro do campo da saúde pública diz respeito, principalmente, à sua forte associação com outras enfermidades crônicas como diabetes melittus tipo 2, doenças coronarianas, hipertensão arterial e hiperlipidemia $^{8}$, pois sabe-se que o risco destas aumenta diretamente com o grau de excesso de peso ${ }^{9} \mathrm{e}$ acúmulo de gordura na região abdominal ${ }^{10}$.

Estudos têm analisado o papel da dieta na gênese das DCNT, especialmente nas doenças cardiovasculares $^{10,11}$. Alguns estudaram o papel das gorduras e dos açúcares simples como fatores de risco para as DCNT, outros o efeito protetor das fibras e antioxidantes para essas doenças $^{12,13}$. Porém, a OMS sugere que a avaliação do consumo alimentar das populações estaria melhor representado pelo padrão alimentar ${ }^{14}$, con- siderando que os indivíduos não consomem nutrientes nem alimentos isoladamente ${ }^{15}$. Nesse sentido, é crescente o interesse nessa linha de investigação sobre o consumo de grupos de alimentos considerados definidores de padrões alimentares saudáveis e não saudáveis ${ }^{2}$.

A caracterização do padrão alimentar, definido por meio de inquéritos de frequência de consumo ${ }^{11,16}$, reflete um retrato geral do consumo habitual dos indivíduos, fornecendo informações importantes sobre a qualidade da dieta, sendo essa caracterização cada vez mais utilizada para analisar a associação entre as características qualitativas da dieta e as doenças crônicas ${ }^{15,17,18}$. Tal abordagem também foi sugerida por Alves et al. ${ }^{14}$, Lenz et al. ${ }^{19} \mathrm{e} \mathrm{Hu}{ }^{20}$, que ressaltaram a importância de se conhecer o padrão alimentar como método mais eficaz para identificar associações entre alimentação, gordura corporal e DCNT.

A questão que se coloca seria até que ponto a pesquisa empírica tem abordado a questão do padrão alimentar como variável preditiva da gordura corporal. Portanto, esta revisão teve como objetivo sistematizar os artigos publicados no período de 7 anos sobre padrões alimentares de risco e sua associação com a gordura corporal, em população adulta.

\section{Métodos}

Nesta revisão, buscaram-se artigos indexados nas bases de dados eletrônicas National Library of Medicine (Medline, USA), Scielo e Literatura Latino-Americana e do Caribe em Ciências da Saúde (Lilacs, Brasil), publicados em língua inglesa e portuguesa entre os anos de 2005 a 2012. Foram utilizados os descritores na língua inglesa: Anthropometry, food consumption, chronic diseases, body fat and adults. Os operadores lógicos and, or e not foram usados para combinar os descritores e termos utilizados na busca dos artigos.

A identificação e a seleção dos estudos foram realizadas pelos pesquisadores, considerando como critérios de inclusão, artigos originais, publicados entre 2005 e 2012, oriundos de estudos transversais com amostras populacionais em adultos, que relacionavam o consumo de alimentos de risco para as DCNT e sua associação com a gordura corporal. Artigos identificados em mais de uma base de dados foram computados em apenas uma.

A qualidade metodológica dos estudos foi avaliada por dois pesquisadores em observância aos seguintes itens: clareza e adequação na des- 
crição do processo de amostragem; aleatorização da amostra; especificação dos critérios de inclusão e exclusão; descrição das perdas e exclusões e apropriada apresentação dos resultados. No caso de discordância entre os pesquisadores, a permanência de um artigo no estudo foi decidida por consenso entre os dois revisores após avaliação dos critérios de inclusão e reanálise do texto. Foram excluídos artigos de revisão e/ou revisão sistemática, estudos do tipo caso-controle, coorte e ensaios clínicos controlados.

\section{Resultados}

Foram localizados 8.924 artigos e, conforme a leitura posterior dos títulos, selecionados 21 para participarem da segunda etapa do estudo. Nessa etapa, os artigos foram reanalisados de forma criteriosa e independente por dois pesquisadores, totalizando 08 artigos para inclusão nessa revisão (Tabela 1 ).

Todos os artigos incluídos nessa revisão foram estudos realizados no Brasil. As principais características dos estudos como autoria, ano de publicação, local de estudo, número de participantes, objetivos do estudo, tipo de coleta e inquérito alimentar, plano de análise e padrões de consumo utilizados estão apresentados no Quadro 1 . Observa-se que 25\% dos estudos não referem qual o questionário de frequência alimentar utilizado (QFA). Nota-se que há diferenças metodológicas entre os estudos concernentes aos aspectos de representatividade da amostra, tipo de QFA e a definição dos padrões de consumo.

O Quadro 2 apresenta um resumo dos resultados encontrados nos estudos selecionados.
Para melhor visualização e comparação entre os resultados, estão sendo apresentados separadamente a exposição (padrões alimentares), o desfecho (excesso de peso e obesidade abdominal) e as associações.

Em Salvador (BA), Oliveira et al. ${ }^{21}$, em um estudo de base populacional, analisaram os fatores associados ao excesso de peso e gordura abdominal em adultos. Na avaliação antropométrica utilizou-se o índice de massa corporal (IMC) e a circunferência da cintura (CC), e para o consumo alimentar QFA não identificado, analisado segundo proposta de Fornés et al. ${ }^{22}$. Os autores encontraram 25,8\% de sobrepeso, $18,8 \%$ de obesidade e $28,1 \%$ de excesso de gordura abdominal. Encontrou-se frequência reduzida de consumo de legumes, frutas e hortaliças (padrão saudável) e elevado consumo de carnes, embutidos, leite e derivados (padrão de risco) em mais de 1/3 da amostra. No modelo final após análise de regressão de Poisson, o padrão alimentar não apresentou associação significante com o excesso de peso (IMC) e gordura abdominal (CC).

Em estudo realizado na cidade de São Paulo, Neumann et al. ${ }^{11}$, identificaram os padrões de consumo alimentar e sua associação com fatores de risco para as doenças cardiovasculares (DCV). Na identificação do excesso de peso foi utilizado o IMC e para obesidade abdominal, a relação cintura-quadril (RCQ), ambos com a classificação da $\mathrm{OMS}^{23,24}$. Quanto ao perfil antropométrico, 48,5\% e 43,1\% apresentaram excesso de peso e obesidade abdominal, respectivamente. O QFA utilizado foi o validado por Ribeiro e Cardoso ${ }^{25}$ para estudo da dieta versus DCNT, dando origem a 4 padrões alimentares: padrão 1 (cafeteria) - leite integral, pães, massas,

Tabela 1. Resultados da pesquisa na literatura.

\begin{tabular}{llc}
\hline \multicolumn{1}{c}{ Base de dados pesquisada } & \multicolumn{1}{c}{ Descritores utilizados } & Número de artigos encontrados \\
\hline Lilacs (descritores) & Food consumption, chronics diseases, & 129 \\
anthropometry, body fat, adults & \\
Medline (Key words) & Food consumption, chronics diseases, & 8.548 \\
Scielo (descritores) & anthropometry, body fat, adults \\
& Food consumption, chronics diseases, & 247 \\
Artigos incluídos para a $2^{\circ}$ fase & Lilacs & \multicolumn{2}{|c}{6} \\
& Medline & 0 \\
Artigos incluídos na revisão & Scielo & 15 \\
& Lilacs & 2 \\
Total de artigos avaliados & Medline & 0 \\
& Scielo & 6
\end{tabular}


Quadro 1. Características dos estudos selecionados entre os anos de 2005 e 2012.

\begin{tabular}{|c|c|c|c|c|c|}
\hline $\begin{array}{c}\text { Autor, ano da } \\
\text { publicação } \\
\text { e local do } \\
\text { estudo }\end{array}$ & Amostra & $\begin{array}{c}\text { Objetivo } \\
\text { do estudo }\end{array}$ & $\begin{array}{c}\text { Tipo de coleta e } \\
\text { inquérito alimentar } \\
\text { utilizado }\end{array}$ & $\begin{array}{c}\text { Plano } \\
\text { de análise }\end{array}$ & $\begin{array}{c}\text { Padrões de } \\
\text { consumo } \\
\text { utilizados }\end{array}$ \\
\hline $\begin{array}{l}\text { Oliveira et } \\
\text { al. }{ }^{21} 2009 . \\
\text { Salvador, BA. }\end{array}$ & 570 & $\begin{array}{l}\text { Identificar os fatores } \\
\text { associados ao excesso } \\
\text { de peso e } \\
\text { concentração de } \\
\text { gordura abdominal } \\
\text { em adultos de ambos } \\
\text { os sexos. }\end{array}$ & $\begin{array}{l}\text { Domiciliar. } \\
\text { Questionário de } \\
\text { frequência alimentar } \\
\text { (sem referência de } \\
\text { qual). }\end{array}$ & $\begin{array}{l}\text { Consumo alimentar: } \\
\text { Escores de frequência de } \\
\text { consumo baseado na } \\
\text { metodologia de Fornés et } \\
\text { al. }^{22} \\
\text { Estimação das razões de } \\
\text { prevalência: regressão de } \\
\text { Poisson. }\end{array}$ & $\begin{array}{l}3 \text { grupos: } \\
\text { Leguminosas, } \\
\text { frutas e hortaliças. } \\
\text { Carnes, } \\
\text { embutidos, leite e } \\
\text { derivados. } \\
\text { Cereais e } \\
\text { derivados. }\end{array}$ \\
\hline $\begin{array}{l}\text { Neumann et } \\
\text { al. }^{11} 2007 . \\
\text { São Paulo, SP. }\end{array}$ & 782 & $\begin{array}{l}\text { Identificar os padrões } \\
\text { de consumo } \\
\text { alimentar e investigar } \\
\text { a associação desses } \\
\text { padrões com fatores } \\
\text { de risco biológicos, } \\
\text { sociodemográficos e } \\
\text { comportamentais } \\
\text { para doenças } \\
\text { cardiovasculares } \\
\text { (DCV). }\end{array}$ & $\begin{array}{l}\text { Domiciliar. } \\
\text { Questionário de } \\
\text { frequência alimentar } \\
\text { semiquantitativo } \\
\text { previamente validado. }{ }^{25}\end{array}$ & $\begin{array}{l}\text { Consumo alimentar: } \\
\text { Escore de frequência de } \\
\text { consumo. } \\
\text { Estimação das razões de } \\
\text { prevalência: Regressão } \\
\text { linear múltipla. }\end{array}$ & $\begin{array}{l}4 \text { tipos: } \\
\text { Cafeteria. } \\
\text { Tradicional. } \\
\text { Moderno. } \\
\text { Aterogênico. }\end{array}$ \\
\hline $\begin{array}{l}\text { Gimeno et } \\
\text { al. }{ }^{17} 2011 . \\
\text { Ribeirão } \\
\text { Preto, SP. }\end{array}$ & 2.197 & $\begin{array}{l}\text { Descrever e } \\
\text { identificar fatores } \\
\text { associados aos } \\
\text { padrões de consumo } \\
\text { de alimentos. }\end{array}$ & $\begin{array}{l}\text { Domiciliar. } \\
\text { Questionário de } \\
\text { frequência alimentar } \\
\text { semi-quantitativo } \\
\text { validado por Fornés et } \\
\text { al. }^{26}\end{array}$ & $\begin{array}{l}\text { Consumo alimentar: } \\
\text { Escores de frequência de } \\
\text { consumo baseado na } \\
\text { metodologia de Fornés et } \\
\text { al. }^{22} \\
\text { Estimação das razões de } \\
\text { prevalência: Regressão de } \\
\text { Poisson. }\end{array}$ & $\begin{array}{l}4 \text { tipos: } \\
\text { Obesogênico. } \\
\text { Saudável. } \\
\text { Misto. } \\
\text { Popular. }\end{array}$ \\
\hline $\begin{array}{l}\text { Perozzo et } \\
\text { al. }{ }^{15} 2008 . \\
\text { São Leopoldo, } \\
\text { RS. }\end{array}$ & 1.026 & $\begin{array}{l}\text { Estudar a associação } \\
\text { de padrões } \\
\text { alimentares com } \\
\text { obesidade geral e } \\
\text { abdominal em } \\
\text { mulheres. }\end{array}$ & $\begin{array}{l}\text { Domiciliar. } \\
\text { Questionário de } \\
\text { frequência alimentar } \\
\text { semiquantitativo } \\
\text { validado por Alves et } \\
\text { al. }^{14}\end{array}$ & $\begin{array}{l}\text { Consumo alimentar: } \\
\text { Análise fatorial baseada } \\
\text { em Alves et al. }{ }^{14} \\
\text { Estimação de razões de } \\
\text { prevalência: Regressão de } \\
\text { Poisson. }\end{array}$ & $\begin{array}{l}5 \text { tipos: } \\
\text { PA-vegetais. } \\
\text { PA-frutas. } \\
\text { PA-nozes/ } \\
\text { oleaginosas. } \\
\text { PA- pão/aipim/ } \\
\text { batata doce. } \\
\text { PA-chocolates/ } \\
\text { doces. }\end{array}$ \\
\hline
\end{tabular}

continua

salgados, doces, gorduras, presunto, refrigerante comum; Padrão 2 (tradicional) - Cereais, feijões, folhosos, não-folhosos e sopas, maionese, chá e café; Padrão 3 (moderno) - Leite desnatado, frutas, sucos naturais, peixes, refrigerantes diet, adoçantes artificiais e, em menor proporção, pães, não folhosos e sopas; Padrão 4 (atero- gênico) - feijoada, carne de boi e miúdos, porco, frango, linguiça, ovo, bebidas alcoólicas e sal. Os padrões cafeteria e aterogênico se associaram positivamente com o excesso de peso e obesidade abdominal, enquanto que os padrões moderno e tradicional se associaram inversamente com o IMC e a RCQ. 


\begin{tabular}{|c|c|c|c|c|c|}
\hline \multicolumn{6}{|c|}{ Quadro 1. continuação } \\
\hline $\begin{array}{c}\text { Autor, ano da } \\
\text { publicação } \\
\text { e local do } \\
\text { estudo }\end{array}$ & Amostra & $\begin{array}{c}\text { Objetivo } \\
\text { do estudo }\end{array}$ & $\begin{array}{c}\text { Tipo de coleta e } \\
\text { inquérito alimentar } \\
\text { utilizado }\end{array}$ & $\begin{array}{c}\text { Plano } \\
\text { de análise }\end{array}$ & $\begin{array}{c}\text { Padrões de } \\
\text { consumo } \\
\text { utilizados }\end{array}$ \\
\hline $\begin{array}{l}\text { Lino et al. }{ }^{27} \\
2011 . \\
\text { Rio Branco, } \\
\text { AC. }\end{array}$ & 1.469 & $\begin{array}{l}\text { Estimar a prevalência } \\
\text { e identificar os } \\
\text { principais fatores } \\
\text { associados ao excesso } \\
\text { de peso em adultos. }\end{array}$ & $\begin{array}{l}\text { Domiciliar. } \\
\text { Questionário de } \\
\text { frequência alimentar } \\
\text { elaborado com base no } \\
\text { questionário aplicado } \\
\text { no Sistema de } \\
\text { Vigilância de Fatores de } \\
\text { Risco e Proteção para } \\
\text { Doenças Crônicas por } \\
\text { Inquérito Telefônico } \\
(\text { VIGITEL) }\end{array}$ & $\begin{array}{l}\text { Consumo alimentar: } \\
\text { categorização do consumo } \\
\text { de frutas e vegetais. } \\
\text { Estimação de razões de } \\
\text { prevalência: Regressão de } \\
\text { Poisson. }\end{array}$ & $\begin{array}{l}4 \text { categorias: } \\
\text { Consumo diário de } \\
\text { frutas - pelo } \\
\text { menos uma vez ao } \\
\text { dia um tipo de } \\
\text { fruta. } \\
\text { Não consumo } \\
\text { diário de frutas. } \\
\text { Consumo diário de } \\
\text { vegetais - pelo } \\
\text { menos um tipo de } \\
\text { legume cozido ou } \\
\text { salada crua todos } \\
\text { os dias. } \\
\text { Não consumo } \\
\text { diário de vegetais. }\end{array}$ \\
\hline $\begin{array}{l}\text { Mondini et } \\
\text { al. }^{29} 2010 . \\
\text { Ribeirão } \\
\text { Preto, SP. }\end{array}$ & 930 & $\begin{array}{l}\text { Avaliar o consumo de } \\
\text { frutas e hortaliças por } \\
\text { adultos e identificar } \\
\text { variáveis } \\
\text { sociodemográficas e } \\
\text { do estilo de vida } \\
\text { associadas ao } \\
\text { consumo desses } \\
\text { alimentos. }\end{array}$ & $\begin{array}{l}\text { Domiciliar. } \\
\text { Questionário } \\
\text { semiquantitativo de } \\
\text { frequência alimentar } \\
\text { validado por Fornés et } \\
\text { al. }^{26}\end{array}$ & $\begin{array}{l}\text { Consumo alimentar: } \\
\text { Escores de frequência de } \\
\text { consumo baseado na } \\
\text { metodologia de Fornés et } \\
\text { al. }^{22} \\
\text { Estimação das razões de } \\
\text { prevalência: Regressão de } \\
\text { Poisson. }\end{array}$ & $\begin{array}{l}2 \text { categorias: } \\
\text { Consumo diário de } \\
\text { frutas e hortaliças. } \\
\text { Consumo mínimo } \\
\text { recomendado de } \\
\text { frutas e hortaliças. }\end{array}$ \\
\hline $\begin{array}{l}\text { Neutzling et } \\
\text { al. }{ }^{30} 2009 . \\
\text { Pelotas, RS. }\end{array}$ & 972 & $\begin{array}{l}\text { Descrever a } \\
\text { frequência de frutas, } \\
\text { legumes e verduras } \\
\text { por adultos de } 20 \text { a } \\
69 \text { anos e analisar os } \\
\text { fatores associados a } \\
\text { tal consumo. }\end{array}$ & $\begin{array}{l}\text { Domiciliar. } \\
\text { Questionário de } \\
\text { frequência alimentar } \\
\text { qualitativo (não } \\
\text { informado qual o tipo). }\end{array}$ & $\begin{array}{l}\text { Consumo alimentar. } \\
\text { Dicotomização das } 5 \\
\text { frequências de consumo } \\
\text { em } 2 \text { categorias e criação } \\
\text { de variável combinando os } \\
3 \text { consumos (frutas, } \\
\text { legumes e verduras). } \\
\text { Estimação das razões de } \\
\text { prevalência: Regressão de } \\
\text { Poisson. }\end{array}$ & $\begin{array}{l}2 \text { categorias: } \\
\text { Consumo regular } \\
\text { de frutas, legumes } \\
\text { e verduras. } \\
\text { Consumo irregular } \\
\text { de frutas, legumes } \\
\text { e verduras. }\end{array}$ \\
\hline $\begin{array}{l}\text { Campos et } \\
\text { al. }{ }^{31} 2010 . \\
\text { Florianópolis, } \\
\text { SC. }\end{array}$ & 1890 & $\begin{array}{l}\text { Estimar a prevalência } \\
\text { do consumo } \\
\text { adequado de frutas, } \\
\text { legumes e verduras e } \\
\text { fatores associados. }\end{array}$ & $\begin{array}{l}\text { Telefônico. } \\
\text { Questionário de } \\
\text { frequência alimentar } \\
\text { elaborado com base no } \\
\text { questionário aplicado } \\
\text { no Sistema Municipal } \\
\text { de Monitoramento de } \\
\text { Fatores de Risco para } \\
\text { Doenças Crônicas Não } \\
\text { Transmissíveis a partir } \\
\text { de entrevistas } \\
\text { telefônicas (SIMTEL) }\end{array}$ & $\begin{array}{l}\text { Consumo alimentar: } \\
\text { Somatória das frequências } \\
\text { diárias de cada grupo } \\
\text { (frutas, legumes e } \\
\text { verduras) para cada } \\
\text { indivíduo e posterior } \\
\text { classificação em adequado } \\
\text { ou inadequado, com base } \\
\text { na recomendação da } \\
\text { OMS } \\
\text { Estimação das razões de } \\
\text { prevalência: Regressão de } \\
\text { Poisson }\end{array}$ & $\begin{array}{l}2 \text { categorias: } \\
\text { Consumo } \\
\text { adequado de } \\
\text { frutas, legumes e } \\
\text { verduras (cinco ou } \\
\text { mais vezes ao dia). } \\
\text { Consumo } \\
\text { inadequado de } \\
\text { frutas, legumes e } \\
\text { verduras. }\end{array}$ \\
\hline
\end{tabular}


Quadro 2. Principais resultados dos estudos selecionados para a revisão.

\begin{tabular}{|c|c|c|c|}
\hline $\begin{array}{c}\text { Autor, ano da } \\
\text { publicação } \\
\text { e local do } \\
\text { estudo }\end{array}$ & $\begin{array}{l}\text { Perfil antropométrico } \\
\text { da amostra }\end{array}$ & Perfil alimentar da amostra & $\begin{array}{c}\text { Associações estatisticamente } \\
\text { significativas }\end{array}$ \\
\hline $\begin{array}{l}\text { Oliveira et } \\
\text { al. }{ }^{21} 2009 . \\
\text { Salvador, BA. }\end{array}$ & $\begin{array}{l}25,8 \% \text { Sobrepeso (IMC) }(26,3 \% \\
\text { mulheres e } 25 \% \text { homens). } \\
12,8 \% \text { Obesidade (IMC) }(15,1 \% \\
\text { mulheres e } 8,4 \% \text { homens). } \\
28,1 \% \text { Excesso de gordura } \\
\text { abdominal (CC) (35,7\% mulheres } \\
\text { e } 12,9 \% \text { homens) }\end{array}$ & $\begin{array}{l}\text { Encontrou-se frequência } \\
\text { reduzida de consumo de } \\
\text { legumes, frutas e hortaliças } \\
\text { (padrão saudável) e elevado } \\
\text { consumo de carnes, } \\
\text { embutidos, leite e derivados } \\
\text { (padrão de risco) em mais de } \\
\text { 1/3 da amostra. }\end{array}$ & $\begin{array}{l}\text { O padrão alimentar não apresentou } \\
\text { associação significante com o excesso } \\
\text { de peso (IMC) e gordura abdominal } \\
\text { (CC). }\end{array}$ \\
\hline $\begin{array}{l}\text { Neumann et } \\
\text { al. }{ }^{11} 2007 . \\
\text { São Paulo, SP. }\end{array}$ & $\begin{array}{l}48,5 \% \text { Excesso de peso (IMC). } \\
43,1 \% \text { Obesidade abdominal } \\
\text { (RCQ). }\end{array}$ & $\begin{array}{l}\text { Não descrimina a } \\
\text { porcentagem da amostra que } \\
\text { apresentou cada padrão. }\end{array}$ & $\begin{array}{l}\text { Os padrões cafeteria e aterogênico se } \\
\text { associaram positivamente com o } \\
\text { excesso de peso e obesidade } \\
\text { abdominal. No entanto, os padrões } \\
\text { moderno e tradicional se associaram } \\
\text { inversamente com o IMC e a RCQ. }\end{array}$ \\
\hline $\begin{array}{l}\text { Gimeno et } \\
\text { al. }{ }^{17} 2011 . \\
\text { Ribeirão } \\
\text { Preto, SP. }\end{array}$ & NÃO INFORMADO. & $\begin{array}{l}\text { O padrão obesogênico foi } \\
\text { mais frequente nos indivíduos } \\
\text { eutróficos }(29,1 \%) \text { e que não } \\
\text { apresentavam obesidade } \\
\text { central }(32,8 \%) \text {.O padrão } \\
\text { saudável foi mais frequente } \\
\text { nos indivíduos com sobrepeso } \\
(29 \%) \text { e obesidade central } \\
(28,1 \%) \text {. }\end{array}$ & $\begin{array}{l}\text { O padrão saudável teve efeito protetor } \\
\text { para obesidade geral e de risco para } \\
\text { obesidade central }(\mathrm{RP}=0,59 \text {; } \\
\mathrm{IC} 95 \% ; 0,42-0,81) \mathrm{O} \text { padrão misto } \\
\text { apresentou efeito protetor para } \\
\text { obesidade geral ( } \mathrm{RP}=0,68 ; \mathrm{IC} 95 \% ; 0,50 \text { - } \\
0,91)\end{array}$ \\
\hline $\begin{array}{l}\text { Perozzo et } \\
\text { al. }{ }^{15} 2008 . \\
\text { São Leopoldo, } \\
\text { RS. }\end{array}$ & $\begin{array}{l}23,3 \% \text { Obesidade abdominal } \\
\text { (CC). } \\
18 \% \text { Obesidade geral (IMC). }\end{array}$ & NÃO INFORMADO. & $\begin{array}{l}\text { Encontrou-se uma associação direta } \\
\text { entre a obesidade geral e o PA- frutas, } \\
\text { embora uma relação inversa com o PA- } \\
\text { vegetais. Já o PA-nozes/oleaginosas } \\
\text { associou-se de forma inversa à } \\
\text { obesidade abdominal. }\end{array}$ \\
\hline
\end{tabular}

Em Ribeirão Preto, estado de São Paulo, Gimeno et al. ${ }^{17}$ descreveram os padrões de consumo de alimentos e fatores associados a estes, entre os participantes de um estudo de base populacional. $\mathrm{Na}$ avaliação antropométrica utilizou-se o IMC e a CC, ambos com a classificação da $\mathrm{OMS}^{24,33}$. Foi utilizado para a avaliação do consumo alimentar, um QFA validado por Fornés et al. ${ }^{26}$, analisado segundo a metodologia de escores proposta por esse mesmo autor ${ }^{22}$, identificandose 4 padrões de consumo de alimentos: Padrão obesogênico - açúcar, doces e refrigerantes; $\mathrm{Pa}$ drão saudável - hortaliças, frutas e laticínios desnatados; Padrão misto - frituras, pescados e raízes e Padrão Popular - feijão, cereais e gordura vegetal. Observou-se maior frequência de indivíduos com padrão "saudável" entre aqueles com excesso de peso e obesidade abdominal, o padrão obesogênico foi mais frequente nos indivíduos eutróficos e que não apresentaram obesidade abdominal, o que poderia ser explicado por uma situação de causalidade reversa. Ou seja, de forma inusitada os padrões obesogênico e misto apresentaram-se como fatores de proteção para a obesidade abdominal avaliada pela CC.

Perozzo et al. ${ }^{15}$, em estudo desenvolvido em São Leopoldo, Rio Grande do Sul, analisaram a associação de padrões alimentares com a obesidade geral e abdominal em mulheres adultas. Para a avaliação antropométrica, foi utilizado o IMC 
Quadro 2. continuação

\begin{tabular}{|c|c|c|c|}
\hline $\begin{array}{l}\text { Autor, ano da } \\
\text { publicação } \\
\text { e local do } \\
\text { estudo }\end{array}$ & $\begin{array}{l}\text { Perfil antropométrico } \\
\text { da amostra }\end{array}$ & Perfil alimentar da amostra & $\begin{array}{c}\text { Associações estatisticamente } \\
\text { significativas }\end{array}$ \\
\hline $\begin{array}{l}\text { Lino et al. } \\
2011 . \\
\text { Rio Branco, } \\
\text { AC. }\end{array}$ & 46,9\% Excesso de peso (IMC). & $\begin{array}{l}58,8 \% \text { Consumo diário de } \\
\text { frutas. } \\
52,4 \% \text { Consumo diário de } \\
\text { vegetais. }\end{array}$ & $\begin{array}{l}\text { Foi encontrada associação } \\
\text { estatisticamente significante entre } \\
\text { consumo diário de frutas e excesso de } \\
\text { peso ente homens ( } \mathrm{RP}=1,19 ; \\
\text { IC95\%;1,02-1,39) e entre as mulheres } \\
(\mathrm{RP}=1,10 ; \mathrm{IC} 95 \% ; 0,96-1,27) \text {. }\end{array}$ \\
\hline $\begin{array}{l}\text { Mondini et } \\
\text { al. }{ }^{29} 2010 . \\
\text { Ribeirão } \\
\text { Preto, SP. }\end{array}$ & NÃO INFORMADO. & $\begin{array}{l}\text { Apenas } 25 \% \text { dos homens e } \\
40 \% \text { das mulheres atenderam } \\
\text { à recomendação mínima de } \\
\text { consumo de frutas e } \\
\text { hortaliças, consumindo esses } \\
\text { alimentos pelo menos cinco } \\
\text { vezes/dia. }\end{array}$ & $\begin{array}{l}\text { O estado nutricional não apresentou } \\
\text { associação com o consumo mínimo } \\
\text { recomendado de frutas e hortaliças ( } \mathrm{p} \\
=0,1441 \text { ) }\end{array}$ \\
\hline $\begin{array}{l}\text { Neutzling et } \\
\text { al. }{ }^{30} 2009 . \\
\text { Pelotas, RS. }\end{array}$ & 51,3\% Excesso de peso (IMC). & $\begin{array}{l}\text { Apenas } 20,9 \% \text { dos indivíduos } \\
\text { consumia frutas, legumes e } \\
\text { verduras regularmente. }\end{array}$ & $\begin{array}{l}\text { O estado nutricional não apresentou } \\
\text { associação estatisticamente significante } \\
\text { com o consumo regular de frutas, } \\
\text { legumes e verduras }(\mathrm{p}=0,76) .\end{array}$ \\
\hline $\begin{array}{l}\text { Campos et } \\
\text { al. }{ }^{31} 2010 . \\
\text { Florianópolis, } \\
\text { SC. }\end{array}$ & NÃO INFORMADO. & $\begin{array}{l}\text { O consumo adequado de } \\
\text { frutas, legumes e verduras } \\
\text { esteve presente em apenas } \\
21,9 \% \text { da amostra. } \\
\text { Frequência maior entre as } \\
\text { mulheres. }\end{array}$ & $\begin{array}{l}\text { Foi encontrada uma associação } \\
\text { significante entre consumo } \\
\text { inadequado de frutas, legumes e } \\
\text { verduras e obesidade geral nos } \\
\text { indivíduos do sexo masculino } \\
(\mathrm{RP}=1,9 ; \text { IC95\%;1,3-2,7). }\end{array}$ \\
\hline
\end{tabular}

na identificação de obesidade geral ${ }^{34}$ e a CC para identificação da obesidade abdominal ${ }^{35}$. Para o consumo alimentar, o QFA utilizado foi o validado por Alves et al. ${ }^{14}$ segundo proposta do mesmo autor, dando origem a 5 padrões alimentares (PA), a partir de uma análise fatorial: PA vegetais - repolho, couve, couve-flor, abóbora, cenoura, laranja, brócolis, pepino, beterraba, tomate, vagem, banana, mamão, maçã, bergamota, outros vegetais verdes e biscoito gelado; PA frutas - melão, melancia, manga, pera, pêssego, caqui, uva, limão, maracujá, abacaxi, sorvete, abacate, goiaba, kiwi, fígado, suco natural e peixes; PA nozes-oleaginosas - Amêndoa, avelã, nozes, castanhas, ameixa seca uva passa, massa integral, veia, farelo de trigo, açúcar mascavo, mel, pão de centeio, pão integral, soja, vinho tinto e arroz integral; PA pão/aipim/batata doce pão caseiro, aipim, batata doce, milho, feijão, lentilha, açúcar, banha, leite integral, batata inglesa, nata, massa e carne de porco; PA chocolates/doces - Chocolates, balas, sobremesas, doces, creme de leite, presunto, mortadela, salame, copa, maionese industrializada, queijo, frituras, fast food, biscoito doce, cuca e bolo. Os autores encontraram $18 \%$ de obesidade geral e $23,3 \%$ de obesidade abdominal. Encontrou-se uma associação direta entre a obesidade geral e o PA frutas, embora uma relação inversa com PA vegetal. O PA nozes/oleaginosas associou-se de forma inversa à obesidade abdominal. Deve-se ressaltar que esses resultados devem ser interpretados com a devida cautela, considerando que a definição dos padrões alimentares utilizada pelos autores foi extremamente heterogênea incluindo em um mesmo padrão alimentos de diversos grupos alimentares.

Em Rio Branco (AC), Lino et al. ${ }^{27}$, em um estudo de base populacional, avaliaram a prevalência e os fatores associados ao excesso de peso 
em adultos. $\mathrm{Na}$ avaliação antropométrica utilizou-se o IMC, segundo a classificação da OMS ${ }^{29}$, e para o consumo alimentar um QFA elaborado com base naquele aplicado no Inquérito de Vigilância de fatores de risco e proteção para doenças crônicas por inquérito telefônico (VIGITEL) ${ }^{28}$, utilizando como variáveis o consumo de frutas e de vegetais. Estas variáveis foram categorizadas em 4 grupos, baseado no consumo de cada indivíduo, assim denominados: Consumidor diário de frutas - indivíduos que consumiam pelo menos uma vez ao dia um tipo de fruta; Consumidor diário de vegetais - consumiam um tipo de legume cozido ou salada crua, pelo menos uma vez ao dia; Não consumidores diários de frutas consumiam frutas em menos de 7 dias na semana e Não consumidores diários de vegetais - consumiam menos de uma vez ao dia legumes cozidos ou salada crua. Quanto ao perfil antropométrico, 46,9\% da amostra apresentaram excesso de peso. $\mathrm{O}$ consumo diário de frutas e vegetais foi de $58,8 \%$ e $52,4 \%$, respectivamente. A análise de regressão de Poisson mostrou uma associação positiva entre consumo diário de frutas e o excesso de peso, em ambos os sexos. É plausível supor que esses resultados configurem mais uma vez a situação de causalidade reversa.

Mondini et al. ${ }^{29}$, em estudo de base populacional, em Ribeirão Preto, estado de São Paulo, avaliaram o consumo de frutas e hortaliças e sua associação com o estado nutricional em adultos. $\mathrm{Na}$ avaliação antropométrica foi utilizado o IMC, com a classificação da $\mathrm{OMS}^{24}$ e para o consumo alimentar QFA validado por Fornés et al. ${ }^{26}$, tendo como período de referência os seis últimos meses que antecederam a entrevista. Analisou-se o QFA segundo proposta de escores deste mesmo autor ${ }^{22}$, originando duas categorias de consumo: Consumo diário de frutas e hortaliças Sim ou não, independente do número de vezes por dia e Consumo mínimo recomendado de frutas e hortaliças - consumo de pelo menos cinco vezes ao dia, baseado no recomendado pela Organização Mundial de Saúde ${ }^{1}$. Encontrou-se elevada frequência de consumo diário de frutas e hortaliças nos dois sexos (mais de 70\%). Considerando a recomendação mínima de consumo diário de frutas e hortaliças, apenas $25 \%$ dos homens e $40 \%$ das mulheres atenderam esta recomendação. A análise de regressão de Poisson, não apontou associação significante entre consumo diário e consumo mínimo recomendado de frutas e hortaliças e o estado antropométrico.

Em estudo de base populacional em Pelotas, no estado do Rio Grande do Sul, Neutzling et al. ${ }^{30}$ estimaram a frequência do consumo de frutas, legumes e verduras em adultos e analisaram os fatores associados a tal consumo. $\mathrm{Na}$ avaliação antropométrica utilizou-se o IMC, classificado segundo critérios da $\mathrm{OMS}^{24}$, e para o consumo alimentar um QFA não identificado, utilizando como variável para análise o consumo de frutas, legumes e verduras nos últimos 12 meses que antecederam a entrevista. Com base nos resultados do QFA, o consumo foi dicotomizado em: Consumo regular de frutas, legumes e verduras - cinco ou mais vezes por semana - ou não. Quanto ao perfil antropométrico, $51,3 \%$ da amostra apresentaram excesso de peso e $20,9 \%$ consumo regular de frutas, legumes e verduras. A análise de regressão de Poisson, não mostrou associação significante entre o consumo regular de frutas, legumes e verduras e o estado nutricional.

Campos et al. ${ }^{31}$ realizaram estudo em Florianópolis (SC), para estimar a prevalência do consumo adequado de frutas, legumes e verduras e fatores associados em adultos participantes do Sistema Municipal de Monitoramento de fatores de risco para doenças crônicas não transmissíveis $\left(\right.$ SIMTEL) ${ }^{32}$ realizado em 2005 . Na avaliação antropométrica foi utilizado o IMC com a classificação da $\mathrm{OMS}^{33}$ e para o consumo alimentar foi utilizado QFA do SIMTEL ${ }^{34}$ sobre consumo de frutas, legumes e verduras. A partir das frequências diárias obtidas no questionário, o consumo de 5 ou mais vezes ao dia de frutas, legumes e verduras foi denominado Consumo adequado, segundo recomendação da $\mathrm{OMS}^{23}$. O consumo esteve adequado em $21,9 \%$ da amostra. Segundo a análise de regressão de Poisson foi encontrada uma associação direta entre consumo inadequado de frutas, legumes e verduras e obesidade nos indivíduos do sexo masculino.

\section{Discussão}

O excesso de peso e a obesidade abdominal têm sido considerados como importantes fatores de risco para o desenvolvimento das DCNT. Atualmente estas representam um problema de grande relevância em Saúde Pública, justificando o crescente número de estudos desenvolvidos nos últimos anos. Diante desse fato, é necessário o conhecimento dos perfis de exposição de risco a essas enfermidades, entre eles o consumo alimentar. Entretanto, no Brasil, observa-se um número extremamente reduzido de estudos abordando o consumo alimentar e a sua associação ao perfil antropométrico. Por sua vez, os resultados desses 
estudos são ainda conflitantes impondo a necessidade de uma revisão integrativa contextualizando as especificidades de cada investigação.

Nos estudos analisados, confirma-se a tendência preocupante do incremento do excesso de peso, cujas prevalências são elevadas em ambos os sexos. Nesse sentido, a prevalência de excesso de peso em adultos variou de $38,6 \%$ a $51,3 \%$ e o excesso de gordura abdominal variou de $23,4 \%$ a $43,1 \%$, valores semelhantes aos encontrados em outros estudos brasileiros ${ }^{36,37}$. Essas cifras estão de acordo com as encontradas no último VIGITEL $^{6}$, realizado em 2011 , no qual 48,5\% da população apresentaram excesso de peso assim como com aquelas divulgadas pela POF 2008$2009^{5}$, onde o excesso de peso foi identificado em $50,1 \%$ dos homens e $48,1 \%$ das mulheres.

As maiores prevalências de excesso de peso foram encontradas nos estudos realizados na região sul do país ${ }^{15,24}$, confirmando os achados da POF 2008-20095, com maiores frequências de excesso de peso nessa região, atingindo 56,8\% dos homens e $51,6 \%$ das mulheres. Salienta-se que alguns estudos aqui incluídos não relataram as prevalências de excesso de peso e de gordura abdominal, dificultando a comparação entre as prevalências encontradas.

Quanto ao consumo alimentar, observa-se na maioria dos estudos a baixa frequência de consumo de alimentos considerados de proteção para DCNT, como é o caso das frutas, legumes e hortaliças.

A variação na forma de construção e na apresentação dos padrões alimentares originou diferenças marcantes quanto ao tipo e número de padrões dificultando sobremaneira a interpretação dos resultados. Nesse sentido, no estudo de Neumann et al. ${ }^{11}$, alimentos considerados de risco para DCNT fizeram parte de diferentes padrões. Por sua vez, no estudo de Perozzo et al. ${ }^{15}$, alimentos considerados de proteção (vegetais e frutas) compuseram padrões de consumo diferentes. Em 50\% dos estudos, utilizou-se como indicador de proteção, o consumo de frutas, legumes e hortaliças, embora apresentando uma ampla variação em sua forma de apresentação: consumo diário ${ }^{29}$, consumo regular ${ }^{23}$, consumo mínimo recomendado ${ }^{31}$, consumo adequado ${ }^{24}$.

A falta de padronização para definição dos padrões de consumo alimentar dificulta sobremaneira a comparação dos resultados obtidos nos diferentes contextos ecológicos. É necessário conhecer melhor a validade e a confiabilidade dos padrões alimentares, pois em métodos de obtenção a posteriori, derivados de análise fatorial ou análise de agrupamento, o investigador toma decisões que podem afetar o número, os tipos de padrões e sua capacidade de predizer doenças crônicas em populações expostas a longos períodos de risco ${ }^{20,38}$.

Nesta revisão, ficam claras as diferenças entre os instrumentos de coleta de dados de consumo alimentar utilizados, prejudicando a interpretação e a comparação dos resultados entre os estudos. Alguns autores relataram a fonte de seus questionários $^{10,15,17,23,31,33}$, outros apenas descrevem as variáveis utilizadas ${ }^{21,24}$. O tempo de referência utilizado no QFA é outro ponto a ser considerado. Dos 08 estudos analisados, 03 não descrevem o tempo utilizado ${ }^{21,31,33}, 01$ empregou o período de 1 mês ${ }^{15}, 02$ o de $6^{17,23}$ e 02 os últimos 12 meses antes da entrevista ${ }^{10,32}$. Essa variação limita a comparação dos estudos, pois os desfechos que possuem diferentes tempos de aparecimento e tempos de referências mais longos propiciam a ocorrência de vieses, como o de memória.

As frequências de consumo de frutas, verduras e hortaliças apresentaram diferenças entre os estudos analisados, com os menores percentuais de consumo encontrados na região sul do país ${ }^{24,33}$, contrariando os achados de Jaime et al. ${ }^{39}$ e do VIGITEL ${ }^{6}$, no qual a cidade de Florianópolis apresentou o maior percentual de consumo recomendado de frutas e hortaliças no Brasil ${ }^{1}$. Esta revisão mostra que o consumo de frutas, legumes e hortaliças pela população brasileira está bem abaixo das recomendações atuais, e vem corroborar os achados de outros estudos ${ }^{39-44}$, inclusive da POF 2008-20095 , nos quais menos de $10 \%$ da população atingem as recomendações de consumo de frutas, verduras e legumes.

O padrão alimentar mostrou-se associado significantemente ao perfil antropométrico em 5 estudos analisados. Destes, em três, o consumo de frutas, verduras e hortaliças mostrou-se diretamente associado com o excesso de peso, o que vem contrariar os resultados de estudos prévios ${ }^{45,46}$.

No estudo de Gimeno et al. ${ }^{17}$, padrões saudáveis representados por consumo adequado de frutas e vegetais, representaram fatores de risco para o excesso de gordura abdominal, contrariando os achados de Castanho et al. ${ }^{47}$ que, ao analisarem o consumo de frutas, legumes e verduras em adultos e sua relação com a síndrome metabólica (SM), observaram que o consumo adequado de frutas apresentou efeito protetor para a obesidade abdominal, hipertrigliceridemia e presença de SM. Esse fato poderia ser explicado pelo viés de informação, uma vez que ao considerar-se o consumo nos últimos seis meses que 
antecederam à entrevista, a possibilidade de viés de memória é potencialmente grande, como também pelo fenômeno da causalidade reversa. $\mathrm{Ou}$ seja, por se tratar de estudo com desenho transversal os indivíduos com excesso de peso poderiam estar em dieta para perda ponderal, mediante a adoção de um padrão alimentar saudável. Os indivíduos também poderiam estar distorcendo o relato de alguns alimentos por já conhecer seu efeito benéfico ou mesmo nocivo ${ }^{14}$.

No estudo de Perozzo et al. ${ }^{15}$, em que frutas e vegetais originaram padrões alimentares diferentes, o elevado consumo de PA - vegetais apresentou-se como fator de risco para obesidade geral, fato também observado em estudo desenvolvido por Sichieri et al. ${ }^{48}$. Esses resultados poderiam ser explicados pela superestimação do consumo de vegetais na dieta dos indivíduos com excesso de peso. Deve-se considerar essa possibilidade ao interpretar os resultados, por tratar-se de um viés de difícil controle na coleta de dados e sem controle na análise.

Por outro lado, os padrões de consumo de alimentos dependem de outros fatores como condições socioeconômicas e demográficas, aspectos culturais ${ }^{49}$ e histórico de vida de cada indivíduo ${ }^{50}$. Além disso, as escolhas alimentares podem se modificar ao longo do tempo, de acordo com a disponibilidade dos alimentos em cada região ${ }^{17}$.

Dos 08 estudos analisados, em apenas $01^{33}$ foi utilizado inquérito telefônico para coleta de dados, realizado pelo SIMTEL ${ }^{34}$ em 2005 na cidade de Florianópolis. O SIMTEL ${ }^{34}$, assim como o VIGITEL ${ }^{6,32}$, apesar de possuir algumas limitações por excluir os indivíduos que residem em domicílios que não possuem telefone fixo, são de grande utilidade devido à sua agilidade, baixo custo e grande representatividade da população.

Deve-se ressaltar que em 02 estudos as informações referentes às estimativas de excesso de peso baseiam-se em peso e altura autorreferidos e não aferidos, não podendo se descartar um potencial viés de informação, com superestimação da altura e subestimação do peso corpo- ral $^{32,33}$. Essa limitação deve ser considerada na interpretação dos dados, podendo ser minimizada como mostrado em alguns estudos ${ }^{51,52}$.

Também é limitante o fato de que os artigos aqui analisados são estudos transversais, os quais estão sujeitos a vieses, como o de causalidade reversa. Apesar disso, estudos transversais de base populacional com amostras representativas são de grande relevância, já que são alternativas rápidas e baratas, sendo muito úteis no dimensionamento dos problemas, criando subsídios para a adoção de ações educativas e promoção de hábitos alimentares saudáveis na população de referência ou em outras com características semelhantes.

\section{Conclusão}

Os efeitos benéficos do consumo de alimentos protetores, como frutas, legumes e hortaliças estão bem estabelecidos na literatura. No entanto, a revisão bibliográfica mostrou resultados conflitantes e que diferem desse conceito pré-estabelecido. Esses resultados inesperados poderiam ser atribuídos ao delineamento metodológico utilizado nos estudos (transversais), à multiplicidade e à inadequada caracterização dos padrões alimentares, além do viés de informação, dentre outros fatores.

Desse modo, as variações encontradas nessa revisão, com um grande número e tipos de padrões alimentares, mostram a necessidade de padronização dessa forma de caracterizar o consumo alimentar para que seja possível uma correta interpretação dos resultados e comparações entre os diferentes estudos.

Além disso, as altas prevalências de excesso de peso e do baixo consumo de frutas, legumes e hortaliças, indicam a necessidade de intervenções, visando à implementação de estratégias de prevenção e promoção da saúde, incentivando a redução do excesso de peso e maior consumo de alimentos ricos em fibras, como frutas e vegetais.

\section{Colaboradores}

ECC Azevedo, AS Diniz, JS Monteiro e PC Cabral participaram igualmente de todas as etapas de elaboração do artigo. 


\section{Referências}

1. World Health Organization (WHO). Diet, nutrition and prevention of chronic diseases. Report FAO/WHO Expert Consulation. Geneva: WHO; 2003. (WHO Technical Report Series, n. 916)

2. Brasil. Ministério da Saúde (MS). Secretaria de Vigilância em Saúde. Departamento de Análise de Situação de Saúde. Plano de ações estratégicas para o enfrentamento das doenças crônicas não transmissíveis (DCNT) no Brasil 2011-2022. Brasília: MS; 2011.

3. World Health Organization (WHO). Preventing chronic diseases: a vital investment. Geneva: WHO; 2005.

4. Lerario DDG, Gimeno SG, Franco LJ, Iunes M, Ferreira SRG, Grupo de estudos em Diabetes na comunidade Nipo-brasileira. Excesso de peso e gordura abdominal para a síndrome metabólica em nipobrasileiros. Rev Saude Publica 2002; 36(1):4-11.

5. Instituto Brasileiro de Geografia e Estatística (IBGE). Pesquisa de Orçamento Familiar (POF) 2008- 2009: Antropometria e estado nutricional de crianças, adolescentes e adultos no Brasil. Rio de Janeiro: IBGE; 2010.

6. Brasil. Ministério da Saúde (MS). Secretaria de Vigilância em Saúde. Secretaria de Gestão Estratégica e Participativa. Vigitel Brasil 2010: vigilância de fatores de risco e proteção para doenças crônicas por inquérito telefônico. Brasília: MS; 2011.

7. Peixoto MRG, Monego ET, Alexandre VP, Souza RGM, Moura EC. Monitoramento por entrevistas telefônicas de fatores de risco para doenças crônicas: experiência de Goiânia, Goiás, Brasil. Cad Saude Publica. 2008; 24(6):1323-1333.

8. Pi-sunyer X. A clinical view of the obesity problem. Science 2003; 299(5608):859-860.

9. World Health Organization (WHO). The World health report 2002: Reducing risks, promoting healthy life. Geneva: WHO; 2002.

10. Petribú MMV, Cabral PC, Arruda IKG. Estado Nutricional, Consumo alimentar e risco cardiovascular: Um estudo em universitários. Rev Nutrição 2009; 22(6):837-846.

11. Neumann AICP, Martins IS, Marcopito LF, Araujo EAC. Padrões alimentares associados a fatores de risco para doenças cardiovasculares entre residentes de um município brasileiro. Rev Panam Salud Publica 2007; 22(5):329-339.

12. De Oliveira MC, Sichieri R, Venturim Mozzer R. A low-energy-dense diet adding fruit reduces weight and energy intake in women. Appetite 2008; 51(2):291295.

13. Sartorelli DS, Franco LJ, Cardoso MA. High intake of fruits and vegetables predicts weight loss in Brazilian overweight adults. Nutr Res 2008; 28(4):233-238.

14. Alves ALS, Olinto MTA, Costa JSD, Bairros FS, Balbinotti MAA. Padrões alimentares de mulheres adultas residentes em área urbana no Sul do Brasil. Rev Saude Publica 2006; 40(5):865-873.

15. Perozzo G, Olinto MTA, Dias-da-costa JS, Henn RL, Sarriera J, Pattussi MP. Associação dos padrões alimentares com obesidade geral e abdominal em mulheres residentes no Sul do Brasil. Cad Saude Publica 2008; 24(10):2427-2439.
16. Fung TT, Willett WC, Stampfer MJ, Manson JE, Hu FB. Dietary patterns and the risk of coronary heart disease in women. Arch Intern Med 2001; 161(15): 1857-1862.

17. Gimeno SGA, Mondini L, Moraes SA, Freitas ICM. Padrões de consumo de alimentos e fatores associados em adultos de ribeirão Preto, São Paulo, Brasil: Projeto OBEDIARP. Cad Saude Publica 2011; 27(3):533-545.

18. Peixoto MRG, Benício MHDA, Jardim PCBV. The relationship between body mass index and lifestyle in a Brazilian adult population: a cross-sectional survey. Cad Saude Publica 2007; 23(11):2694-2704.

19. Lenz A, Olinto MTA, Dias-da-costa JS, Alves AL, Balbinotti M, Pattussi MP, Bassani DG. Socioeconomic, demographic and lifestyle factors associated with dietary patterns of women living in Southern Brazil. Cad Saude Publica 2009; 25(6):1297-1306.

20. Hu FB. Dietary pattern analysis: a new direction in nutritional epidemiology. Curr Opin Lipidol 2002; 13(1):3-9.

21. Oliveira LPM, Assis AMO, Silva MCM, Santana MLP, Santos NS, Pinheiro SMC, Barreto ML, Souza CO. Fatores associados a excesso de peso e concentração de gordura abdominal em adultos na cidade de Salvador, Bahia, Brasil. Cad Saude Publica 2009; 25(3):570-582.

22. Fornés NS, Martins IS, Velasquez-melendez G, Latorre MRDO. Escores de consumo alimentar e níveis lipêmicos em população de São Paulo, Brasil. Rev Saude Publica 2002; 36(1):12-18.

23. World Health Organization (WHO). Global strategy on diet, physical activity and health. Geneva: WHO; 2004.

24. World Health Organization (WHO). Physical status: The use and interpretation of anthropometry. Geneva: World Health Organization: 1995. (WHO technical Report Series, 854).

25. Ribeiro AB, Cardoso MA. Construção de um questionário de frequência alimentar como subsídio para programas de prevenção de doenças crônicas não transmissíveis. Rev Nutr 2002; 15(2):239-245.

26. Fornés NS, Stringhini MLF, Elias BM. Reproducibility and validity of food frequency questionnaire for use among low-income Brazilian workers. Public Health Nutr 2003; 6(8):821-827.

27. Lino MZR, Muniz PT, Siqueira KS. Prevalência e fatores associados ao excesso de peso em adultos: inquérito populacional em Rio Branco, Acre, Brasil, 2007-2008. Cad Saude Publica 2011; 27(4):797810.

28. Brasil. Ministério da Saúde (MS). Secretaria de Vigilância em Saúde (Vigitel). Vigitel Brasil 2006. Vigilância de fatores de risco e proteção para doenças crônicas por inquérito telefônico. Brasília: MS; 2007.

29. Mondini L, Moraes AS, Freitas ICM, Gimeno SGA. Consumo de frutas e hortaliças por adultos em Ribeirão Preto, SP. Rev Saude Publica 2010; 44(4):686694. 
30. Neutzling MB, Rombaldi AJ, Azevedo MR, Hallal PC. Fatores associados ao consumo de frutas, legumes e verduras em adultos de uma cidade no Sul do Brasil. Cad Saude Publica 2009; 25(11):2365-2374.

31. Campos VC, Bastos JL, Gauche H, Boing AF, Assis MAA. Fatores associados ao consumo adequado de frutas, legumes e verduras em adultos de Florianópolis. Rev Bras Epidemiol 2010; 13(2):352-362.

32. Monteiro CA, Moura EC, Jaime PC, Lucca A, Florindo AA, Figueiredo ICR, Bernal R, Silva NN. Monitoramento de fatores de risco para doenças crônicas por entrevistas telefônicas. Rev Saude Publica 2005; 39(1):47-57.

33. World Health Organization (WHO). Obesity: preventing and managing the global epidemic. Geneva: WHO; 1997. (Report of WHO Consultation on Obesity)

34. World Health Organization (WHO). Obesity: preventing and managing the global epidemic. Geneva: WHO; 2000. Report of a WHO consultation on obesity.

35. Lean MEJ, Han TS, Morrison CE. Waist circumference as a measure for indicating need for weight management. BMJ 1995; 311(6998):158-161.

36. Moura EC, Morais Neto OL, Malta DC, Moura L, Silva NN, Bernal R, Claro RM, Monteiro CA. Vigilância de Fatores de Risco para Doenças Crônicas por Inquérito Telefônico nas capitais dos 26 estados brasileiros e no Distrito Federal (2006). Rev Bras Epidemiol 2008; 11(1):20-37.

37. Yokota RTC, Vasconcelos TF, Ito MK, Dutra ES, Baiocchi KC, Merchán- Hamann E, Lopes EB, Barbosa RB. Prevalência de fatores de risco para doenças crônicas não-transmissíveis em duas regiões do Distrito Federal. Comun Ciênc Saúde 2007; 18(4): 289-296.

38. Moeller SM, Reedy J, Millen AE, Dixon LB, Newby PK, Tucker KL, Krebs-Smith SM, Guenther PM. Dietary patterns: challenges and opportunities in dietary patterns research. J Am Diet Assoc 2007; 107(7):1233-1239.

39. Jaime PC, Figueiredo ICR, Moura EC, Malta DC. Fatores associados ao consumo de frutas e hortaliças no Brasil, 2006. Rev Saude Publica 2009; 43(Supl. 2):57-64.

40. Jaime PC, Monteiro CA. Fruit and vegetable intake by Brazilian adults, 2003. Cad Saude Publica 2005; 21(Supl.):19-24.

41. Figueiredo ICR, Jaime PC, Monteiro CA. Fatores associados ao consumo de frutas, legumes e verduras em adultos na cidade de São Paulo. Rev Saude Publica 2008; 42(5):777-785.

42. Jansen AK, Lopes ACS, Lopes filho JD. Fatores associados ao excesso de peso em funcionáriosde um hospital universitário. Rev Baiana de Saúde Pública 2010; 34(4):951-966.
43. Levy RB, Claro RM, Mondini L, Sichieri R, Monteiro CA. Distribuição regional e socioeconômica da disponibilidade domiciliar de aliementos no Brasil em 2008-2009. Rev Saude Publica 2012; 46(1):6-15.

44. Carvalho EO, Rocha EF. Consumo alimentar de população adulta residente em área rural da cidade de Ibatiba (ES, Brasil). Cien Saude Colet 2011; 16(1):179-185.

45. Trudeau E, Kristal AR, Li S, Patterson RE. Demographic and psychosocial predictors of fruit and vegetable intakes differ: Implications for dietary interventions. J Am Diet Assoc 1998; 98(12):1412-1417.

46. Kristal AR, Hedderson MM, Patterson RE, Neuhauser ML. Predictors of self-initiated, healthful dietary changes. J Am Diet Assoc 2001; 101(7):762766.

47. Castanho GKF, Marsola FC, Mclellan KCP, Nicola M, Moreto F, Burini RC. Consumo de frutas, verduras e legumes associado à Síndrome Metabólica e seus componentes em amostra populacional adulta. Cien Saude Colet 2013; 18(2):385-392.

48. Sichieri R, Castro JFG, Moura AS. Fatores associados ao padrão de consumo alimentar da população brasileira urbana. Cad Saude Publica 2003; 19(Sup. 1):S47-S53.

49. Whichelow MJ, Prevost AT. Dietary patters and their association with demographic, lifestyle and health variables in a random sample of British adults. Blr J Nutr 1996; 76(1):17-30.

50. Palma RFM, Barbieri P, Damião R, Poletto J, Chaim R, Ferreira SRG, Sartorelli DS. Fatores associados ao consumo de frutas, verduras e legumes em nipobrasileiros. Rev Bras de Epidemiol 2009; 12(3):436445.

51. Peixoto MRG, Benício MHD, Jardim PCBV. Validity of self-reported weight and height: the Goiânia study, Brazil. Rev Saude Publica 2006; 40(6):1065-1072.

52. Lucca A, Moura EC. Validity and reliability of selfreported weight, height and body mass index from telephone interviews. Cad Saude Publica 2010; 26(1): 110-122.

Artigo apresentado em 19/08/2013

Aprovado em 25/10/2013

Versão final apresentada em 30/10/2013 